\title{
Non-linear SHS phenomena: Experiment, theory, numerical modeling
}

\author{
B.V.Novozhilov \\ Institute of Chemical Physics, USSR Academy of Sciences, \\ 117334, Moscow
}

\begin{abstract}
Besides being of technological importance, the SHS processes are also very interesting from the scientific standpoint. For gasless and filtration combustion it was shown in the linear approximation that instability of steady-state regimes with multidimensional perturbations arises more readily than in the one-dimensional case. As a result beyond the scope of the linear approximation the theory predicts a wide spectrum of very peculiar nonsteady burning regimes such as oscillatory combustion or spin waves. In the last twenty years many problems involving in steady-state and nonstationary SHS flame propagation have been investigated both theoretically and experimentally. The main objective of this presentation is to provide a review of recent developments in nonsteady non-linear SHS phenomena. The following topics are considered: experimental investigation of the non-steady regimes, stability of steady-state regimes, nonsteady autowave phenomena and their classification, and the possibility of chaotic behavior. Analytical and numerical results pertaining to these problems are discussed in brief. Experimental results available in the literature are compared with those inferred from analytical and/or numerical studies. It is concluded that the theoretical results cannot be regarded as quite satisfactory for the explanation of information obtained from experimental studies. Areas of future studies are also discussed.
\end{abstract}

\section{INTRODUCTION}

In an experimental study, Merzhanov, Filonenko and Borovinskaya (refs.1,2) reported on new nonstationary phenomena in the combustion of SHS systems. Two ordered modes self-oscillatory combustion and spinning combustion have been discovered and investigated. These interesting phenomena are related to the loss of stability of steady-state propagation and to the study on the combustion modes in the instability region.

Over the past decades, several causes of instability in flames have been found, including heat conduction and diffusion processes responsible for the structure of the flame front. It is remarkable that as early as 1934, Lewis and Elbe (ref.3) focused their attention on the so-called enthalpy excess in the combustion wave in prefixed gaseous systems. For a clear understanding of the combustion wave stability the concept of the enthalpy excess is of great importance. When the thermal diffusivity $\kappa$ exceeds the diffusion coefficient $D$ there is a maximum in the total enthalpy in the preheating zone, and the flame may be unstable. The flame front absorbs the excess energy into itself and thereby enters a generally unstable state. The Lewis number in a gas $L e=D / K$ is of the order of unity, thus the enthalpy excess in the gas flame can not be large. In contrast with the flame in a gas, the enthalpy excess is especially large in the limit case which the condensed medium.

This idea was fully confirmed by Zeldovich in a study of the nonsteady combustion of solid propellants (ref.4). He showed that in considering sufficiently slow nonsteady processes one should take into account solely the ther mal inertia of the condensed phase, which is described by the heat conduction equation. Calculations within the framework of the model have shown that the stability of the propellant combustion wave is determined only by the enthalpy excess of the fuel and the sensitivity of the burning rate to variations in the initial temperature. The combustion wave is aperiodically unstable with the product of these quantities greater than the critical one. 
Zeldovich's theory of nonsteady burning of propellants, which assumes the constancy of its surface temperature, served as a basis for the development of a more general theory accounting for the variability of the surface temperature (refs.5,6). The steady-state burning criterion in the propellant model with a variable surface temperature accounting for only the inertia of the heated layer of the condensed phase is expressed through two parameters, which characterize the variations in burning rate and surface temperature with initial temperature. At the stability boundary the real part of the frequency is equal to zero. As to the imaginary part of the frequency, it is non-zero at and near the stability boundary. Thus, beyond the stability boundary, an oscillatory regime with a growing amplitude exists to a linear approximation.

A propellant is an oscillatory system with an infinite number of degrees of freedom; besides the burning rate, the temperature also oscillates at every point of the condensed phase. The mathematical statement of the problem differs in this case from that for linear and nonlinear oscillations of mechanical and electrical systems. Instead of second-order differential equations with nonlinear terms to which most of the problems on oscillations of systems with a finite number of degrees of freedom are reducible, we have to investigate a nonlinear partial differential equation (refs.6,7). For not-very-high amplitudes of free oscillations the governing equation of such a system can be solved by the method of successive approximations.

As was demonstrated in (ref.7) beyond the scope of the linear approximation the theory predicts very peculiar burning regimes: self-sustained oscillations at a constant pressure and hysteresis, resonance phenomena at sub- and super-harmonics at an oscillatory pressure. Thus, in the paper cited it was first shown analytically that the self-oscillating flame front in solid fuel arises as bifurcation from a solution describing a plane steady-state front.

The gasless auto-oscillatory burning was first discovered and studied by Shkadinsky, Khaikin and Merzhanov (ref.8) via a numerical solution for the nonsteady-state problem of the combustion wave onset in the condensed media.

Several analytical and numerical studies were undertaken in order to shed some light on the facts about both one- and two-dimensional instabilities in gasless system (refs.9-11). For flameless and gasless burning it was shown that instability in the systems with two-dimensional perturbations arises more readily than in the one-dimensional case (refs.6,9).

It must be noted that, as early as 1950, a pulsation mode was observed during combustion of thermites which could have been of an oscillatory nature, however, the authors gave no detailed investigations (ref.12).

An appearance of the papers cited in the beginning of the chapter (refs.1,2) has stimulated in many respects the fast development of nonsteady SHS systems studies. A short review is given here to provide readers with a picture for the experimental, analytical and numerical occurs with the condensed medium.

This idea was fully confirmed by Zeldovich in a study of the nonsteady combustion of solid propellants (ref.4). He showed that in considering sufficiently slow nonsteady processes one should take into account solely the thermal inertia of the condensed phase, which is described by the heat conduction equation. Calculations within the framework of the model have shown that the stability of the propellant combustion wave is determined only by the enthalpy excess of the fuel and the sensitivity of the burning rate to variations in the initial temperature. The combustion wave is aperiodically unstable with the product of these quantities greater than the critical one.

Zeldovich's theory of nonsteady burning of propellants, which assumes the constancy of its surface temperature, served as a basis for the development of a more general theory accounting for the variability of the surface temperature (refs.5,6). The steady-state burning criterion in the propellant model with a variable surface temperature accounting for only the inertia of the heated layer of the condensed phase is expressed through two parameters, which characterize the variations in burning rate and surface temperature with initial temperature. At the stability boundary the real part of the frequency is equal to zero. As to the imaginary part of the frequency, it is non-zero at and near the stability boundary. Thus, beyond the stability boundary, an oscillatory regime with a growing amplitude exists to a linear approximation. 
A propellant is an oscillatory system with an infinite number of degrees of freedom; besides the burning rate, the temperature also oscillates at every point of the condensed phase. The mathematical statement of the problem differs in this case from that for linear and nonlinear oscillations of mechanical and electrical systems. Instead of second-order differential equations with nonlinear terms to which most of the problems on oscillations of systems with a finite number of degrees of freedom are reducible, we have to investigate a nonlinear partial differential equation (refs.6,7). For not-very-high amplitudes of free oscillations the governing equation of such a system can be solved by the method of successive approximations.

As was demonstrated in (ref.7) beyond the scope of the linear approximation the theory predicts very peculiar burning regimes: self-sustained oscillations at a constant pressure and hysteresis, resonance phenomena at sub- and super-harmonics at an oscillatory pressure. Thus, in the paper cited it was first shown analytically that the self-oscillating flame front in solid fuel arises as bifurcation from a solution describing a plane steady-state front.

The gasless auto-oscillatory burning was first discovered and studied by Shkadinsky, Khaikin and Merzhanov (ref.8) via a numerical solution for the nonsteady-state problem of the combustion wave onset in the condensed media.

Several analytical and numerical studies were undertaken in order to shed some light on the facts about both one- and two-dimensional instabilities in gasless system (refs.9-11). For flameless and gasless burning it was shown that instability in the systems with two-dimensional perturbations arises more readily than in the one-dimensional case (refs.6,9).

It must be noted that, as early as 1950, a pulsation mode was observed during combustion of thermites which could have been of an oscillatory nature, however, the authors gave no detailed investigations (ref.12).

An appearance of the papers cited in the beginning of the chapter (refs.1,2) has stimulated in many respects the fast development of nonsteady SHS systems studies. A short review is given here to provide readers with a picture for the experimental, analytical and numerical results obtained in this field of the combustion science.

The attention of readers is also directed to several comprehensive review papers including those of Merzhanov (refs.13-15) and Margolis (ref.16). The current understanding of the theory of combustion waves in homogeneous media is presented in an excellent paper by Merzhanov and Khaikin (ref.17). An extensive review of the nonlinear macroscopic effects is given by Merzhanov and Rumanov (ref.18). The description of nonsteady phenomena in other combustible systems (such as solid propellants or premixed gases) is beyond the scope of the present paper. Extensive bibliographies are available in the works of Novozhilov (refs.6,19), Margolis and Williams (ref.20) and Clavin (ref.21).

\section{EXPERIMENT}

This review does not purport to give a detailed analysis of the enormous amount of experimental material or a comprehensive bibliographical coverage; it is solely concerned with the basic experimental results obtained over the last few years.

Filtration combustion systems (refs.1, 2, 22-24) and gasless combustion systems have been investigated. The gasless systems included both powder mixtures of transition metals with non-metals (refs.1, 2, 22, 25-31) and different thermite compositions (refs.23, 32-40).

As a rule, cylindrical samples were used, however, some experiments were carried out with laminated (refs.30, 34-36) and other samples (ref.34).

The following modes were observed under different experimental conditions:

C - stationary regime. A combustion reaction being initiated locally by an outer source may further propagate throughout the substance at a constant rate and occur in the narrow zone which separated an initial substance and reaction products.

$P$ - pulsating regime. The phenomenon exists due to the fact that under certain conditions periodic oscillations of combustion rate are exited spontaneously, and the combustion front moves in a pulsating regime. The regime may be either planar or nonplanar. 
S - spinning combustion. At ignition of the sample a glowing spot of small diameter appears on its side surface, moves in a spiral along the side surface of an unburnt part of the sample from the site of its appearance to the end of combustion process, and leaves behind a bright trace which gradually merges with a similar trace from previous turn. The spinning combustion regimes were found both in the porous metallic sample-gaseous nitrogen system and in the gasless combustion. In the first case, the sample burns along the side surface; the inner part of the sample either does not burn up at all, or reacts completely after the surface combustion front has passed. In the second case, it was over the volume (a circular motion of the burning plane around the cylinder axis).

M - multispot combustion (disordered modes) - aperiodic oscillation of the combustion rate, random migration of luminous spots in the combustion front.

L - limit burning regime. The limit burning regime is characterized by the periodical arising of the (hotbed) seat on the sample sides, by their bifurcation with the following moving (motion) to the edges, by the merging on edges and the galloping down along them.

The possible realization of different modes depends on the sample composition, the amount of inert diluent, the initial temperature of the sample, the amount of heat abstraction into the ambient medium, the density and sizes of element particles of the initial mixture, pressure of the inert or reacting gas, and other factors. It can be observed in one and the same system under varied parameters being various manifestations of unstable combustion.

The sequence of the mode variation under changing different parameters is of great interest. Some papers are specially devoted to the elucidation of the modes order and different methods of combustion condition changing were used (ref.39,40). The experimental procedure applied made it possible to change smoothly the process condition in order to observe the regularities of non-steady-state alternation of different combustion modes.

As a result authors come to the conclusion that the succession of modes alternation could be presented as a rule as follows: C-P-M-S-L. It is noted that sometimes some modes are fallen out of this succession. Note as well that there are exclusions to the rule mentioned above. So it is announced (ref.40) that steady-state burning regime just transfers to the spin combustion at small cross-section sample sizes.

\section{THEORY}

The phenomena described above are usually associated with internal instability of a plane steady-state flame front which prevents its lasting a long time. It will be noted, however, that loss of stability of the steady-state wave may be soft, when a regime with a finite amplitude is excited due to an accidental slight perturbation with the desired frequency, and rigid, when a regime can be obtained only by exerting a vigorous action on the system. Rigid excitation evidently occurs in a stable (to a linear approximation) burning regime, and soft excitation in an unstable regime.

The question of the stability of a stationary combustion regime of condensed systems with respect to deformations of the flame front has been raised by Makhviladze and this author (ref.9). Since a full analytical solution of this problem is not possible the approximation of an infinitely thin chemical conversion zone adopted for the first time in connection with investigations on the question of the diffusely thermal stability of a laminar gas flame was employed (ref.42).

This assumption permits us to consider the zone of chemical transformation of the initial substance as narrow compared with the width of the preheating zone, and regard it as a surface separating the initial substance from the reaction products. As a result, the problem is incomplete and, therefore, the approximation of an infinitely narrow reaction zone necessitates a "closure" hypothesis for the rate of unsteady-state burning.

Further on we will assume that the linear burning rate $u$ depends exclusively on the temperature in the chemical transformation zone $T_{b}$. Then the variation in burning rate with temperature can be described, to a linear approximation, by the coefficient

$$
k=\left(T_{b}^{0}-T_{a}\right) \mathrm{d} \ln u^{0} / \mathrm{d} T_{b}^{0}
$$

whose explicit expression depends on the type of steady-state burning law $u^{0}\left(T_{b}^{0}\right)$. Here $T_{a}$ is the initial temperature, and the quantities pertaining to the steady combustion regime 
are labelled with a zero superscript. In this form, the results of the stability study must have a wider applicability than only for the case of the Arrhenius dependence of the reaction rate on temperature.

In the case of the Arrhenius dependence of the chemical reaction rate, we have, neglecting the power dependence as compared to the exponential one,

$$
u_{\sim}^{0} \exp \left(-E / R T_{b}^{0}\right) \text { and } k=\left(T_{b}^{0}-T_{a}\right) E / R\left(T_{b}^{0}\right)^{2}
$$

A perturbation theory analysis of the stability of the stationary states gives the following result for the stability boundary (ref.9) If $\lambda$ denotes the wave length of the perturbation and $s=4 \pi \kappa / \lambda u^{\circ}$ - the nondimensional wave number then the steady-state regime is stable at $k<k^{*}$ where the stability boundary $k^{*}=F(s)$ can be expressed by

$$
F(s)=\left(4+3 s^{2}+\sqrt{\left(4+3 s^{2}\right)^{2}+4\left(1+s^{2}\right)^{3}}\right) / 2\left(1+s^{2}\right)
$$

The frequency at the stability boundary is purely imaginary, i.e. the stability loss is of an oscillatory nature. As a function of the perturbation wavelength, it is expressed thus:

$$
\omega^{*}=\left(1+s^{2}\right) k^{*}(s) / 4
$$

Assuming $s=0$ in Eq.3 , we find that the region of instability forone-dimensional perturbations is located at $k>k^{*}(0)$, where

$$
k^{*}(0)>2+\sqrt{5} \approx 4.24 \text {. }
$$

In ref.8. Shkadinsky, Khaikin, and Merzhanov made a numerical study of the burning stability of gasless compositions in one-dimensional formulation. It is interesting to compare the numerical calculations accounting for the front width of the exothermal reaction with the value of $k^{*}(0)$ obtained under the assumption that $E / R T_{b}^{0} \gg 1$. The boundary separating the steady-state burning zone from the oscillatory burning zone is determined, according to ref. 8 , by the relation $\alpha=1$, where

$$
\alpha=\left[9.1 T_{b}^{0} /\left(T_{b}^{0}-T_{a}\right)-2.5\right] E / R T_{b}^{0}
$$

This interpolation formula represents the results of computerized calculations. At $\alpha>1$, steady-state burning is stable. If $\alpha<1$, burning is oscillatory.

Having in mind Eq.2, one can find from Eq.6 the value of $k^{*}(0)$ at which one-dimensional stability is lost:

$$
k^{*}(0)=4.55\left(1+2.5 R T_{b}^{0} / E\right)^{-1}
$$

The value $k^{*}(0)=4.24$ obtained under the assumption of an infinitely narrow chemical reaction zone agrees fairly well with this expression.

The minimum value of the parameter characterizing stability, $k_{\min }^{*}=4$, is attained for $s=1$. This indicates that two-dimensional perturbations may prove less stable than one-dimensional. Within the framework of the adopted approximation, however, this conclusion is not rigorous enough. Indeed, the analysis neglected the temperature variation in the reaction zone $\Delta T$, which is in the order of magnitude $\mathrm{R} T^{2} / E$, as compared with the characteristic temperature range $T_{b}^{0}-T_{a}$. Thus, the value $\Delta T /\left(T_{b}^{0}-T_{a}\right) 1 / k$ was considered small. Near the stability boundary $k \approx 4$, therefore, the error introduced into the calculation is of the order of ten per cent. At the same time, the decrease in parameter $k^{*}$ with the variation of $s$ from zero to unity is only six per cent. Consequently, the inference about the presence of a minimum on the curve $k^{*}(s)$ is not rigorous - the effect is of the order of the error permissible in calculation (refs.6,43). 
The model of an infinite narrow reaction zone has been used to find the stability boundary of gasless systems burning taken into account the heat loss (refs.9,11,44), component melting (refs.45-47) and complex chemical kinetics (refs.48-50).

We would note that attempts have been made to improve analytically the approximation of an infinitely narrow reaction zone. Thus, a matching condition in the chemical reaction zone has been proposed (ref.51) that is different to that given in the paper cited (ref.9): the combustion rate is considered to depend not only on the temperature, as is normally done in the narrow zone method, but on the temperature gradients in the matching zone. It was unjustifiably stated (ref.51) that the inertia of the chemical conversion zone is taken into account, since the equations of the conservation of matter and energy in this zone were not considered.

This author and co-workers (refs.52,53) examined, using numerical computation, the question of the stability of a stationary combustion regime of a gasless composition, taken into account the finite thickness of the reaction zone. The cases of various order chemical reactions and a broad zone were studied, and the stability limit was found in the linear approximation. It is shown that two-dimensional perturbations are really more dangerous than one-dimensional those. Interpolation formulae have been given for relating the parameters of the system to the wavelength of the perturbation at the stability limit and for the frequency of the perturbations.

In a typical experiment, a combustion wave propagates through a long sample of finite transverse dimensions and the boundary conditions discretize the admissible transverse wave numbers. We consider, for example, the case of an infinitely long strip with periodic condition at its boundaries. Such a problem arises for a surface filtration combustion (refs.54,55).

The values of the admissible wave numbers depend on the width $L_{\mathrm{m}}$ of the strip and can be obtained from a discretized version of Eq.3: $\quad F\left(s_{\mathrm{m}}\right)$ with $s=4 \pi \mathrm{mK} / \mathrm{Lu}{ }^{0}$.

The sequence of separate combustion modes exited is defined so by the ratio $\mathrm{K} / \mathrm{Lu}^{\circ}$. If $L \ll \kappa / u^{0}$ we have $s_{m} \gg 1$ and at the beginning $P$ - regime is arisen. If pointed ration is small then first of all modes those with large wave number are exited. The references 56-62 consider the analysis of sequence of separate combustion modes.

To describe the nonsteady modes of combustion on the unstable side of the neutral stability boundary requires a nonlinear analysis to calculate the finite amplitudes. Rather a great number of papers is devoted to the searching of the considering problem with the help of bifurcation theory (refs.56-60,63-65). Naturally, it is used there a simplified combustion modes with infinity thin reaction zone. The difficulty mentioned above and connected with non high preciseness of results, that is inherent to this approximation, is not taken into account. Sometimes, the question of discovering stability boundary is not put at all in some investigations (refs.57-59), that is why the results obtained there do not permit simple interpretation.

Bifurcation theory is concerned with the determination of the amplitudes of perturbations only in the vicinity the neutral stability boundary. There is but one study (ref.66) where a pronounced non-linear regime of pulsating combustion was treated analytically. Using integral equation for non-stationary burning velocity (ref.67), the authors considered self-oscillating combustion regime as a sequence of substance heating and fast burning of a layer having a rather high temperature.

A phenomenological theory of nonstationary combustion waves was discussed by Zeldovich and co-workers (refs.68-72). The theory is based on a phenomenological equation describing wave front motion provided the reaction zone being suggested infinitely thin, a well known fact of oscillatory instability for stationary plane waves, and existence of local heat-conductive bond between neighboring elements of the front surface. The case of two-dimensional surface combustion is considered and it is demonstrated that different nonstationary front propagation regimes may exist depending on the size of the surface and its topological properties. One of such regimes is spin combustion of a cylindrical surface when the reaction nidus moves along a spiral. It is difficult to comment these works because the authors said that "the phenomenological equation is not a consistent realization of any asymptotic of exact combustion equations and may claim only qualitative description of two-dimensional nonstationary combustion." 


\section{NUMERICAL MODELING}

Due to the complexity of the problem of nonlinear phenomena in the burning rate of SHS systems any further progress in its solution can only be achieved by using numerical methods. The first step in this direction was made by Merzhanov and co-workers (ref.8) who, in one-dimensional formulation, considered the burning of gasless systems. A study was made into the propagation of a nonsteady front of an exothermal reaction, which is described by two differential equations (a heat conduction equation and an equation of the chemical balance).

Equations to describe the combustion wave unsteady-state propagation in the condensed medium are of the form:

$$
\begin{aligned}
& \frac{\partial T}{\partial t}=\kappa \frac{\partial^{2} T}{\partial x^{2}}+\frac{Q}{c_{p}} k_{0}(1-\eta) \exp (-E / R T) \\
& \frac{\partial \eta}{\partial t}=k_{0}(1-\eta) \exp (-E / R T) \\
& t=0(x>0) \text { and } x \Rightarrow \infty: T=T_{a}, \eta=0 ; \\
& x=0(t>0): T=T_{b} .
\end{aligned}
$$

For simplicity, Eqs.8 are written for a first-order reaction and constant thermophysical characteristics of the medium. According to Eqs.8, the combustion wave in the medium is initiated by a heated surface located at $x=0$ and maintained at a temperature $T_{b}>T_{a}$.

The combustion wave propagation velocity (unsteady-state or steady-state) was determined in numerical calculations as the velocity of spatial motion of the reaction rate maximum.

An analysis of the numerical solution of the problem posed indicated the existence of two qualitatively different front propagation regimes. In the first regime, after the effect of the heated wall diminishes, a steady-state burning process sets in: the front propagation rate is independent of time ( $C$ - regime). In the second regime the front propagation rate does not tend to a definite limit in the course of time, but oscillates about a certain value (P - regime).

Near the stability limit burning rate oscillations are almost sinusoidal, while their amplitude is small. Receding from the stability limit the oscillations acquire properties inherent in relaxation oscillations, a single cycle exhibiting several surges and depressions, i.e. depression of the combustion wave speed is followed by considerable bursts. Close to the stability boundary, the mean velocity of the auto-oscillatory burning is about the normal combustion velocity As the distance to the stability boundary is increased, the mean velocity of auto-oscillator burning monotonously falls.

The same problem was also considered (ref, 73)in the case of a broad reaction zone. In the cited papers higher-order transition to multiply-periodic pulsation were observed. As the stability boundary is moving away, the oscillation width (after the unsteady-state wave covers the distance of the oscillation width the process is repeated) first somewhat grows and then is about doubled in a narrow range of governing parameters. Exactly this type of propagation was observed in the experimental study of the auto-oscillatory burning (ref.27).

The combustion velocity oscillations become more and more complicated as the distance to the stability boundary of the steady-state regime grows (refs.74-78). It should be mentioned, that the authors were not able to detect multiple period of oscillations higher than eight. Beyond the limit line for period eight very complex oscillatory patterns were observed. These patterns did not show any kind of periodicity for very long times of integration and can be identify as chaotic. In order to evaluate the scenery of bifurcations presented Feigenbaum's universal constants have been calculated (ref.76).

The similar behavior of the front propagation in condensed phase combustion employing a model, which takes into account melting effects (ref.74) was observed. More recent calculations (ref.77) showed that periodic behavior may be interrupted by irregular bursts 
(intermittence). The time between successive bursts was found to be random and decreased until ultimately the solution became fully chaotic.

All these direct calculations were one-dimensional and thus, as discussed above, only apply to samples of sufficiently small transverse dimensions. For more realistic sample sizes fully three-dimensional calculations are required.

A mathematical model of the spin burning has been suggested and numerically realized by Shkadinsky and co-workers (refs.54,55,79). It was assumed that a condensed-phase first-order exothermic reaction occurs on the cylindrical surface. $C_{-}, P_{-}$, and $S-$ modes of burning have been obtained. Spin burning has been numerically obtained in the instability region of the steady-state regime with the cylinder diameter not too small, i.e. in that region of the parameters where the auto-oscillatory burning appears in a one-dimensional statement of the problem. As the cylinder diameter grows while other conditions remain the same, two centers of the chemical reaction, instead of one, appear (a double-center spin). Conversely, with the cylinder diameter decreasing, burning became auto-oscillatory. This problem for cylindrical and laminated samples was also worked out (refs.60-62).

It can be seen that spin burning has been numerically realized (refs.54,54) with very small diameters (smaller than $1 \mathrm{~mm}$ ). Real experiments are run with specimens of a much larger diameter. Hence, the problems of spinning combustion at real diameter values cannot be regarded as completely resolved.

It can be asserted, at any rate, that spatial instability in condensed systems burning is an important and interesting problem which waits further numerical study.

\section{DISCUSSION}

It follows from the presented review of investigations on non-linear effects of SHS combustion systems that thermal theory of nonsteady burning allows to explain qualitatively many phenomena observed in experiments. First of all let us denote the existing boundary in the frames of this theory of stability of steady-state combustion regime to one- and multidimensional small disturbances. The theory is able to explain qualitatively the most part of nonsteady combustion modes recorded in experiments and in some cases - their stability as well. In spite of the affirmation about the strict order alternation of modes in experiments with the changing controlling parameters (refs.39,40) it follows from the theory that this order is connected with the relation between cross-section of the sample and internal characteristic size of the preheated zone. In any case analytical and numerical solutions made in one dimensional approximation have the definite sense for small cross-section sample sizes.

Let us denote that any theoretical approaches to explain a plane spiral burning (ref.41) are absent. We would like to point in this connection large external similarity of this kind of burning with well known Lizigang's rings and spirals arisen in the process of weakly soluble sediment formation with chemical reactions (ref.80).

The preceding brief review of nonlinear phenomena indicates that theoretical results cannot currently be compared quantitatively with those experimental. This is due, first of all, to the fact that in analytical and numerical investigations the mechanism chosen to simulate burning process was oversimplified. Almost in all theoretical studies the reaction zone was assumed infinitely narrow, and the errors inherent in this approximation were disregarded. The overwhelming majority of computations were performed for one-step reactions with narrow zones (consideration of one-step reaction with a wide zone is less common). Meanwhile, experimental investigations of steady-state regimes of gasless burning points to extreme complexity of the structure of chemical conversion zones. In this context it is worth mentioning the thermocouple investigations by Zenin and co-workers (refs.81,82), which demonstrated that narrow reaction zones hardly ever exist in burning of gasless systems. In most cases multi-zone conversion (up to four spatially-separated chemical reaction zones) is observed, which include also physical processes, such as melting and capillary spread.

Apart from that, any analytical or numerical investigation implies that the thermophysical parameters of the system (density, heat capacity, thermal conductivity, etc.) and their dependencies on particle size, conversion degree, temperature, etc.,are given. With few exceptions (ref.83) the aforementioned quantities are usually supposed constant in analytical and numerical modeling, which,surely, does not conform to reality. As regards the experimental data on thermophysical parameters, they are extremely meager (ref.84). 
Consider the prospects of application of analytical and numerical methods to studying nonlinear nonstationary phenomena occurring during the SHS systems burning. In most cases these processes prove to be strongly nonlinear, i.e. they originate and proceed far away from stability boundary of a flat stationary flame front. It suffices to recall, e.g., a bright isolated spot of chemical reaction observed in the spinning burning mode. At the same time the problem is liable to analytical solution only in the vicinity of the stability boundary, where the departure of the burning regime from the steady-state is considered a small correction. Even with such simplification the derivation of analytical expressions is extremely cumbersome. Investigates applying analytical approach call for elaboration of computer programs that would facilitate algebraic manipulation.

As indicated above, numerical modeling of SHS processes started with formulation of one-dimensional problems, which was motivated by the drawbacks of the early electronic computers. Making the dynamics of pulsations more complicated places heavy demands on the accuracy of computations and increases the computer time consumption. Certain difficulties arise when passing to the solution of multidimensional problems. It cannot have been a matter of chance that all multidimensional problems formulated thus far were intended to simulate the burning of the specimens with small transverse dimensions, which insures that oscillations with have low wave numbers.

The aforesaid sounds somewhat pessimistic; however it is safe to say that the beauty of the phenomena described in the review and appreciable potentialities of mathematics and computer design with provide a further progress in this area.

\section{REFERENCES}

1. A.G.Merzhanov, A.K.Filonenko and I.P.Borovinskaya, Dokl. Akad. Nauk SSSR, 208, 892-894 (1973).

2. A.G.Merzhanov and I.P.Borovinskaya, Combust. Sci. Technol., 10, 195-201 (1975).

3. B.Lewis and G.Elbe, J.Chem.Phys. 2, 537-547 (1934).

4. Ya.B.Zeldovich, Zhurn.Eksper,i Teoret.Fiziki, 12, 498-524 (1942).

5. B.V.Novozhilov, Zhurn.Prikl.Mekhaniki i Tekhn.Fiziki, No.4,157-160 (1965).

6. B.V.Novozhilov, Nestatsionarnoe Gorenie Tverdykh Raketnykh Topliv, Izd-vo Nauka, Moscow, (1973). (Nonsteady Burning Solid Rocket Propellants, Translation AFSC FTD-MT-24-317-74).

7. B.V.Novozhilov, Zhurn.Prikl.Mekhaniki i Tekhn.Fiziki, No.5, 31-41 (1966).

8. K.G.Shkadinsky, B.I.Khaikin and A.G.Merzhanov, Fiz. Goren. Vzryva., No.1, 19-28 (1971).

9. G.M.Mackviladze and B.V.Novozhilov, Zhurn.Prikl.Mekhaniki i Tekhn. Fiziki, No.5, 51-59 (1971).

10. E.I.Maksimov and K.G.Shkadinsky, Fiz. Goren. Vzryva, No.3, 454-458 (1971).

11. K.G.Shkadinsky and B.I Khaikin, Gorenie i Vzryv, Nauka, Moscow, 104-109 (1972).

12. A.F.Belyaev and L.D.Komkova, Zhurn. Fiz. Khimii, 24, 1302-1311 (1950). 13. A.G.Merzhanov, Archwm Procesow Spalania 5, 17-39 (1974).

14. A.G.Merzhanov, Archs_Combust. 1, 23-48 (1981).

15. A.G.Merzhanov, Proc. of Joint Meeting of the Soviet and Italian Sections of the Combustion Institute, Pisa, plenary lecture, (1990).

16. S.B.Margolis, Prog.Energy Combust.Sci. 17, 135-162 (1991).

17. A.G.Merzhanov and B.I.Khaikin, Prog.Energy Combust.Sci. 14, 1-98 (1988).

18. A.G.Merzhanov and E.N.Rumanov, Uspechi Fiz.Nauk, 151, 553-593 (1987).

19. B.V.Novozhilov, Proc, of Joint Meeting of the Soviet and Italian Sections of the Combustion Institute, Pisa, paper 7.1, (1990).

20. S.B.Margolis and F.A.Williams, Combust. Sci. Technol., 22, 143-169 (1980).

21. P.Claven, Prog. Energy Combust.Sci. 11, 1-59 (1985).

22. A.K.Filonenko, In Collected Edition: Combustion processes in chemical technology and metallurgy (edited by A.G.Merzhanov). Chernogolovka, 258-273 (1975).

23. A.K.Filonenko and V.I.Vershinnikov, Fiz. Goren. Vzryva, No.3, 353-362 (1975).

24. A.K.Filonenko, Archiwum termodynamiki i spalania 6 , No.1, 23-35 (1975).

25. I.P.Borovinskaya, A.G.Merzhanov, N.P.Novikov and A.K.Filonenko, Fiz. Goren. Vzryva, No.1, 4-15 (1974).

26. M.Shkiro and I.P.Borovinskaya, In Collected Edition: Combustion processes in chemical technology and metallurgy (edited by A.G.Merzhanov).Chernogolovka, 253-258 (1975).

27. V.M.Shkiro and G.A.Nersisyan, Fiz. Goren. Vzryva., No.1, 149-151 (1978).

28. V.M.Shkiro, G.A.Nersisyan and I.P.Borovinskaya, Fiz. Goren. Vzryva, No.4, $58-64$ (1978).

29. Y.M.Maksimov, A.T.Pak, G.V.Lavrenchook, Y.S.Nayborodenko and A.G.Merzhanov, Fiz. Goren. Vzryva., No.3,156-159 (1979).

30. Y.M.Maksimov, A.G.Merzhanov, A.T.Pak and M.N.Kuchkin, Fiz. Goren. Vzryva, No.4, 51-58 (1981). 
31. V.M.Shkiro, Proc. of Joint Meeting of the Soviet and Italian Sections of the Combustion Institute, Pisa, paper 4.2 (1990).

32. V.I.Ermakov, A.G.Strunina and V.V. Barzykin, Fiz. Goren. Vzryva, No.2, 211-217 (1976).

33. A.G.Strunina and A.V.Dvoryankin, Dokl. Akad. Nauk SSSR, 260, No.5, 1185-1188 (1981).

34. A.V.Dvoryankin, A.G.Strunina and A.G.Merzhanov, Fiz. Goren. Vzryva., No.2, 10-16 (1982).

35. A.G.Strunina, A.V.Dvoryankin and A.G.Merzhanov, Fiz. Goren. Vzryva., No.2, $30-36$ (1983).

36. V.A.Volpert, A.V.Dvoryankin, A.G.Strunina and A.G.Merzhanov, Fiz. Goren. Vzryva, No.4, 10-13 (1983).

37. A.V.Dvoryankin, A.G.Strunina and A.G.Merzhanov, Fiz. Goren. Vzryva., No.4, 44-47 (1985).

38. A.G.Strunina, E.A.Butakova, L.K.Demidova and V.V.Barzykin, Fiz. Goren. Vzryva, No.2, 99-102 (1988).

39. V.V.Barzykin and A.G.Strunina, Proc.of Joint Meeting of the Soviet and Italian Sections of the Combustion Institute, Pisa, paper 4.5 (1990).

40. A.V.Dvoryankin and A.G.Strunina, Fiz. Goren. Vzryva, No.2, 41-46 (1991).

41. A.G.Merzhanov, A.V.Dvoryankin and A.G.Strunina, Dokl. Akad. Nauk SSSR, 267,869-872 (1982).42. G.I.Barenblatt, Ya.B.Zeldovich and A.G.Istratov, Zhurn.Prikl. Mekhaniki i Tekhn.Fiziki, No.4, 21-26 (1962).

43. G.M.Mackviladze and B.V.Novozhilov, Collected Edition, Teplo - Massoperenos, Minsk, 2, 2 (1972).

44. A.P.Aldushin and S.G.Kasparjan, Fiz. Goren. Vzryva, No.2, 74-77 (1981).

45. S.B.Margolis, SIAM J.appl.Math. 43, 351-369 (1983).

46. S.B.Margolis, Combust.Sci.Technol. 43, 197-215 (1985).

47. A.P.Aldushin, V.A.Volpert and V.P.Filipenko, Fiz. Goren. Vzryva, No.4, 35-41 (1987).

48. A.P.Aldushin and S.G.Kasparjan, Khim. Fiz., 1, 1412-1420 (1981).

49. V.A.Volpert and P.M.Krishenik, Fiz. Goren. Vzryva, No.2, 24-32 (1986).

50. V.A.Volpert and P.M.Krishenik, Fiz. Goren. Vzryva, No.3, 31-38 (1986).

51. A.P.Aldushin and S.G.Kasparjan, Dokl. Akad. Nauk SSSR, 244, 67-70 (1979).

52. O.A.Borisova, V.B.Lidskii, M.N.Neigauz and B.V.Novozhilov, Khim. Fiz., 5, 822-830 (1986).

53. O.A.Borisova, V.B.Lidskil, M.N.Neigauz and B.V.Novozhilov, Fiz. Goren. Vzryva, No.1, 81-84 (1990).

54. T.P.Ivleva, A.G.Merzhanov and K.G.Shkadinsky, Dokl. Akad. Nauk SSSR. 239, 1086-1088 (1978).

55. T.P.Ivleva, A.G.Merzhanov and K.G.Shkadinsky, Fiz. Goren. Vzryva., No.2, 3-10 (1980).

56. Sivashinsky G.I., SIAM J. Appl.Math., 40, 432-438 (1981).

57. V.A.Volpert, A.I.Volpert and A.G.Merzhanov, Dokl. Akad. Nauk SSSR, 262, $642-645$ (1982)

58. V.A.Volpert, A.I.Volpert and A.G.Merzhanov, Dokl. Akad. Nauk SSSR, 263, $918-921$ (1982).

59. V.A.Volpert, A.I.Volpert and A.G.Merzhanov, Fiz. Goren. Vzryva, No.4, 69-72 (1983).

60. S.P.Radev and S.B.Shcherbak, Dokl. Akad. Nauk Bulgaria, 35, 475-479 (1982).

61. S.B.Shcherbak, Fiz. Goren. Vzryva., No.5, 9-12 (1983).

62. S.B.Shcherbak, Fiz. Goren. Vzryva., No.2, 23-27 (1984).

63. B.J.Matkowsky and G.I.Sivashinsky, SIAM J.appl.Math. 35, 465-478 (1978)

64. S.B.Margolis, H.G.Kaper, G.K.Leaf and B.J.Matkowsky, Combust.Sci. Technol., 43, $127-165$ (1985).

65. M.R.Booty, S.B.Margolis and B.J.Matkowsky, SIAM J.appl.Math.,46, 801-843 (1986)

66. V.B.Librovich and G.M.Mackviladze, Zhurn.Prikl.Mekhaniki i Tekhn. Fiziki, No.6, $107-116$ (1974).

67. B.V.Novozhilov, Zhurn.Prikl.Mekhaniki i Tekhn.Fiziki, No.4, 73-78 (1970).

68. A.P.Aldushin, Ya.B.Zeldovich and B.A.Malomed, Dokl. Akad. Nauk SSSR, 251, 1102-1106 (1980). 69. A.P.Aldushin and B.A.Malomed, Fiz. Goren. Vzryva, No.1, 3-12 (1981).

70. A.P.Aldushin, B.A.Malomed and Ya.B.Zeldovich, Comb. Flame,42, No.1, 1-6 (1981).

71. B.A.Malomed, Dokl. Akad. Nauk SSSR, 256, 1383-1387 (1981).

72. D.V.Strunin, Fiz. Goren. Vzryva, No.5, 28-33 (1990).

73. A.P.Aldushin, T.M.Martemjanova, A.G.Merzhanov, B.I.Khaikin and K.G.Shkadinsky, Fiz. Goren. Vzryva., No.5, 613-626 (1973).

74. A.Bayliss and B.J.Matkowsky, J.comp.Phys. 71, 147-168 (1987).

75. A.Bayliss, B.J.Matkowsky and M.Minkoff, SIAM J.appl.Math. 49, 1047-1063 (1989).

76. P.Dimitriou, J.Puszynski and V.Hlavacek, Combust.Sci.Technol. 68, 101-111 (1989)

77. A.Bayliss and B.J.Matkowsky, SIAM J.appl.Math. 50, 437-459 (1990).

78. D.V.Strunin, Zhurn. Vych. Mat. i Matem. Fiz., 31, 543-550 (1991).

79. T.P.Ivleva and K.G.Shkadinsky, Fiz. Goren. Vzryva., No.1, 138-140 (1981).

80. F.M.Schemyakin and P.F.Michalev,Fiziko-Khimitcheskie Perioditcheskie Prozessy, Iz-dvo AN SSSR (1938).

81. A.A.Zenin, A.G.Merzhanov and G.A.Nersisyan, Fiz. Goren. Vzryva, No.1 79-86 (1981).

82. A.A.Zenin, Pure and Appl.Chem. 62, 889-897 (1990).

83. A.P.Aldushin and B.I.Khaikin, Fiz. Goren. Vzryva, No.1, 128-130 (1975).

84. E.A.Butakova and A.G.Strunina, Fiz. Goren. Vzrvva., No.1, 71-73 (1985). 